\title{
Pacific
}

Journal of

Mathematics

\section{THE ANOSOV THEOREM FOR EXPONENTIAL SOLVMANIFOLDS}

EDWARD KePPELMANN AND CHRISTOPHER K. MCCORD 


\title{
THE ANOSOV THEOREM FOR EXPONENTIAL SOLVMANIFOLDS
}

\author{
Edward C. Keppelmann and Christopher K. MCCord
}

\begin{abstract}
A well-known lower bound for the number of fixed points of a self-map $f: X \rightarrow X$ is the Nielsen number $N(f)$. Unfortunately, the Nielsen number is difficult to calculate. The Lefschetz number $L(f)$, on the other hand, is readily computable, but usually does not estimate the number of fixed points. It is known that $N(f)=|L(f)|$ for all maps on nilmanifolds (homogeneous spaces of nilpotent Lie groups) and that $N(f) \geq|L(f)|$ for all maps on solvmanifolds (homogeneous spaces of solvable Lie groups). Typically, though, the strict inequality holds, so the Nielsen number cannot be completely computed from the Lefschetz number. In the present work, we produce a large class of solvmanifolds for which $N(f)=|L(f)|$ for all self maps. This class includes exponential solvmanifolds: solvmanifolds for which the corresponding exponential map is surjective. Our methods provide Nielsen and Lefschetz number product theorems for the Mostow fibrations of these solvmanifolds, even though the maps on the fibers in general will belong to varying homotopy classes.
\end{abstract}

\section{Introduction.}

If $f: M \rightarrow M$ is a map on a compact manifold, the Nielsen number $N(f)$ provides a lower bound on the number of fixed points of any map in the homotopy class of $f$. The classical Lefschetz fixed point theorem says that if the Lefschetz number $L(f) \neq 0$ then any map homotopic to $f$ must have a fixed point. Thus in so far as it is computable, the Nielsen number improves this result, establishing of a minimum number of fixed points, not just the existence of fixed points, for every map homotopic to $f$. However, the Nielsen number has the disadvantage that it is not as readily computable as the Lefschetz number. Thus relations between the two numbers, which allow the increased information of the Nielsen number to be combined with the greater computability of the Lefschetz number, are of great interest.

In 1975 Brooks, Brown, Pak and Taylor [7] showed that $N(f)=|L(f)|$ for any map $f: T \rightarrow T$ on a torus. This theorem was extended to self maps on nilmanifolds (homogeneous spaces of nilpotent Lie groups) by Anosov [5] 
and Fadell-Husseini [8] in 1985-86. We refer to results of this type, which establish equalities between Nielsen and Lefschetz numbers of maps, as Anosov theorems. In 1991, McCord [14] showed that $N(f) \geq|L(f)|$ for all self maps on solvmanifolds (homogeneous spaces of solvable Lie groups), and conjectured that the equality $N(f)=|L(f)|$ would hold for all self maps on a solvmanifold if and only if that solvmanifold was in fact a nilmanifold. In this paper we disprove this conjecture by extending the Anosov theorem to a large class of solvmanifolds. This class, which we label as $\mathcal{N} R$-solvmanifolds, includes all exponential solvmanifolds. The techniques used involve an analysis of the nonorientiable fibration, known as the Mostow fibration, for these solvmanifolds. This fibration has the important property that every homotopy class contains a map which is fiber preserving, but the nonorientability of the fibration forces us to make a very careful study of the relationship between the maps on the fibers over different fixed point classes of the base map. Our methods actually provide a Nielsen and Lefschetz number product theorem for these fibrations, even though the maps on the fibers over the different fixed points classes in the base are in different homotopy classes.

Throughout this paper $G$ will denote a connected simply connected solvable Lie group. Each element $g \in G$ induces the inner automorphism on $G$ of conjugation by $g$, which in turn induces a Lie algebra automorphism $\operatorname{Ad}(g)$ on the Lie algebra $L(G)$ of $G$. Let $\mathcal{E}$ denote the class of all such $G$ with the property that no $\operatorname{Ad}(g)$ has an eigenvalue other than one of unit modulus. Auslander [2] shows that this is equivalent to being an exponential solvable Lie group: a solvable Lie group such that the exponential map $\exp : L(G) \rightarrow G$ is surjective. A solvmanifold $S=\tilde{S} / \Delta$ is called exponential if $\tilde{S}$ is an exponential Lie group. We introduce here a class of solvmanifolds which contains exponential solvmanifolds, and which we denote as $\mathcal{N} R$-solvmanifolds. While precisely defined in the next section, it can be heuristically understood as consisting of solvmanifolds $S=G / \Delta$ such that no $\operatorname{Ad}(g)$ contains a root of unity as an eigenvalue other than one itself. The main result of this paper is:

Theorem 1. If $f: S \rightarrow S$ is a self map of a compact $\mathcal{N} R$-solvmanifold, then $N(f)=|L(f)|$.

As a source of examples consider the 3 dimensional special solvmanifolds $[3,12]$. These have the form $S=J / \Delta$ where $J=\mathbb{R}^{2} \times_{\psi} \mathbb{R}$ is the semidirect product of $\mathbb{R}$ acting on $\mathbb{R}^{2}$ by a one parameter subgroup $\psi$ of $S L_{2}(\mathbb{R})$ and $\Delta$ is the integer lattice in $\mathbb{R}^{3}$. Since $\Delta$ is a subgroup we have that $\psi(1) \in S L_{2}(\mathbb{Z})$. By a change of coordinates it is possible to assume, without change to the homeomorphism type of $S$, that the eigenvalues of $\psi(1)$ are either positive real or complex roots of unity. In the first case we have an 
exponential solvmanifold while in the second we have what is called a rotational solvmanifold. All of these 3 dimensional examples have a Mostow fibration which fibers $S$ over a circle with two dimensional torus as fiber.

The Klein bottle $K$ is an example of a 2 dimensional solvmanifold which is not special (i.e. $K$ cannot be written as the coset space of a connected simply connected solvable Lie group modulo a discrete subgroup). $K$ can be represented as $W / \Theta$ where $W=\mathbb{C} \times{ }_{\sigma} \mathbb{R}$ with $\sigma(t) z=e^{2 \pi i t} z$ and

$$
\Theta=\left\{\left(n+i y, \frac{m}{2}\right) \mid n, m \in \mathbb{Z}, y \in \mathbb{R}\right\} .
$$

Now $\left(0, \frac{1}{2}\right)$ has an adjoint action with eigenvalue -1 and in fact it is shown in $[\mathbf{5}, \mathbf{1 4}]$ that $K$ has self maps $f$ with $|L(f)|<N(f)$. Likewise, all of the three dimensional rotational solvmanifolds admit self maps with $N(f)>|L(f)|$. That is, the Anosov theorem is not true for all solvmanifolds.

The paper can be outlined as follows. Section 2 will describe the topology of nilmanifolds and solvmanifolds, and develop the factorization of self maps made possible by the Mostow fibration. In section 3, we present a brief review of general Nielsen theory and the fibration theory considerations needed in the proof of theorem 1 , thereby reducing the problem to a question in linear algebra. The matrix analysis needed to complete the proof is then carried out in section 4 .

\section{Solvmanifolds and Nilmanifolds.}

In this section, we describe the relevant topological properties of nilmanifolds and solvmanifolds. The topology of nilmanifolds is thoroughly analyzed by Mal'cev [13]; solvmanifolds by Mostow and Auslander[1, 2, 17].

Suppose that $G$ is a connected, simply connected nilpotent Lie group. Let $\Gamma \subset G$ be a discrete uniform subgroup of $G$. Then $N=G / \Gamma$ is a nilmanifold whose universal cover is $G$ and whose fundamental group can be identified with $\Gamma$. While the definition of nilmanifold is more general than this, every nilmanifold has a representation of this form [13]. Since $G$ is simply connected nilpotent, the exponential map exp : $L(G) \rightarrow G$ is a homeomorphism. Thus, as in the case of tori, $N$ is aspherical, and homotopy classes of self maps on $N$ are in one to one correspondence with endomorphisms of $\Gamma$. Further, any endomorphism on $\Gamma$ extends uniquely to an endomorphism of $G[\mathbf{8}, \mathbf{1 3}]$, so every homotopy class of self maps on $N$ contains a unique map induced by an endomorphism $F: G \rightarrow G$ with $F(\Gamma) \subseteq \Gamma$.

Because $G$ is nilpotent the descending central series of commutators $G=$ $G_{1} \supseteq G_{2} \supseteq \ldots$, where $G_{i+1}=\left[G, G_{i}\right]$, terminates with some $G_{k}=\{1\}$. Let 
$\Gamma_{i}=\Gamma \cap G_{i}$, and let $\Lambda_{i}=\Gamma_{i-1} / \Gamma_{i}$. The relevant properties of these groups are summarized in the following

Proposition 2.1. If $G$ is a connected simply connected nilpotent Lie group, $\Gamma \subset G$ a discrete uniform subgroup, then the series $\Gamma_{i}$ and $\Lambda_{i}$ have the following properties:

1. $\Gamma_{i}$ is a uniform subgroup of $G_{i}$.

2. $\left[\Gamma, \Gamma_{i}\right]$ is a subgroup of finite index in $\Gamma_{i+1}$.

3. $\Lambda_{i}$ is torsion-free abelian.

4. Each $\Gamma_{i}$ is a fully invariant subgroup of $\Gamma$.

5. $\Gamma$ acts trivially on each $\Lambda_{i}$.

Proof. The first assertion is proved in [13]. The second and the third each follow immediately in turn. To prove the fourth assertion, let $\phi$ be an endomorphism of $\Gamma$, and let $F: G \rightarrow G$ be the extension of $\phi$ to $G$. Then $F\left(G_{i}\right) \subset G_{i}$, so $\phi\left(\Gamma_{i}\right) \subset \Gamma_{i}$.

Since each $\Gamma_{i}$ is fully invariant in $\Gamma$, it is normal in $\Gamma$. That is, if $g \in \Gamma$ and $c_{g}: \Gamma \rightarrow \Gamma$ is conjugation by $g$, then $c_{g}\left(\Gamma_{i}\right) \subset \Gamma_{i}$. Thus there is a well-defined induced map $\bar{c}_{g i}: \Lambda_{i+1} \rightarrow \Lambda_{i+1}$. The assertion is that, $\bar{c}_{g i}=i d$ for all $i$. To see this, let $h \in \Gamma_{i}$. Then $g h^{-1} g^{-1} h \in \Gamma_{i+1}$, or $g h g^{-1} \Gamma_{i+1}=h \Gamma_{i+1}$.

The significance of this is that all of these algebraic constructions and properties have topological realizations. As each $G$ is itself a connected simply connected nilpotent Lie group, $N_{i}=G_{i} / \Gamma_{i}$ is a compact nilmanifold with fundamental group $\Gamma_{i}$. The quotient $G_{i} / G_{i+1}$ is a connected, simply connected abelian Lie group, with $\Lambda_{i+1}=\Gamma_{i} / \Gamma_{i+1}$ a uniform subgroup, so

$$
T_{i+1}=\left[G_{i} / \Gamma_{i}\right] /\left[G_{i+1} / \Gamma_{i+1}\right] \cong\left[G_{i} / G_{i+1}\right] / \Lambda_{i+1}
$$

is a torus with fundamental group $\Lambda_{i+1}$. The fibration $G_{i+1} \rightarrow G_{i} \rightarrow G_{i} / G_{i+1}$ induces a fibration $N_{i+1} \rightarrow N_{i} \rightarrow T_{i+1}$. These are all aspherical, and there is an isomorphism of short exact sequences

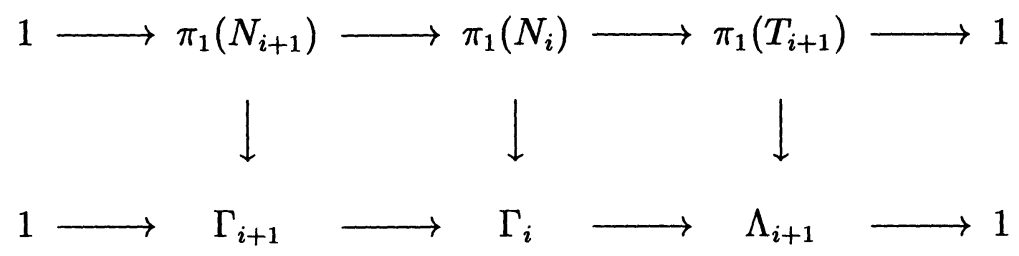

If $f: N \rightarrow N$ is a self map on $N$, we may assume (up to homotopy) that $f$ is given by an endomorphism $F: G \rightarrow G$. This endomorphism then 
induces endomorphisms on $G_{i}$ and maps $f_{i}: N_{i} \rightarrow N_{i}$. These in turn define endomorphisms $F_{i}: \Lambda_{i+1} \rightarrow \Lambda_{i+1}$ and maps $\bar{f}_{i+1}: T_{i+1} \rightarrow T_{i+1}$. Clearly, there are commutative diagrams

$$
\begin{gathered}
N_{i+1} \longrightarrow N_{i} \longrightarrow T_{i+1} \\
\downarrow_{f_{i+1}} \quad \downarrow^{f_{i}} \longrightarrow{ }^{\bar{f}_{i+1}} \\
N_{i+1} \longrightarrow N_{i} \longrightarrow T_{i+1}
\end{gathered}
$$

The collection of maps $\left\{F_{i}\right\}$ will be referred to as the linearization of $f: N \rightarrow$ $N$. The homotopy type of $f$ is not uniquely determined by its linearization, but we will see that, for the purposes of Nielsen theory, the linearization carries sufficient information about $f$.

In comparison with nilmanifolds, every compact solvmanifold can be represented as the coset space $S=G / \Delta$ of a connected, simply connected solvable Lie group $G$ by a uniform closed subgroup $\Delta$. Both the similarities and the differences in the two constructions will be of interest to us. Properties common to solvmanifolds and nilmanifolds are:

1. The fundamental group $\pi=\pi_{1}(S)$ is a torsion-free finitely generated group, and is represented by $\Delta / \Delta_{0}$, where $\Delta_{0}$ is the connected component of the identity in $\Delta$.

2. $S$ is a compact aspherical manifold, and in particular, a $K(\pi, 1)$.

3. Two such manifolds are homeomorphic if and only if their fundamental groups are isomorphic.

4. There is a one-to-one correspondence between homotopy classes of self-maps $[S, S]$ and endomorphisms $\operatorname{Hom}(\pi, \pi)$.

A strongly torsion-free $S$-group is a group $\pi$ with a finitely generated torsion-free nilpotent normal subgroup $\Gamma \triangleleft \pi$ such that $\pi / \Gamma$ is free abelian. Clearly, such a group is solvable, but not every finitely generated torsion-free solvable group is a strongly torsion-free $S$-group. A group $\pi$ can be realized as the fundamental group of a nilmanifold if and only if $\pi$ is torsion-free finitely generated nilpotent. It can be realized as the fundamental group of a solvmanifold if and only if it is a discrete strongly torsion-free $S$-group.

The differences between nilmanifolds and solvmanifolds will be equally important. The essential difference, from which all others follow, is that the exponential map exp : $L(G) \rightarrow G$ is a surjection if $G$ is nilpotent (and a homeomorphism if $G$ is simply connected), but may not be surjective if $G$ is solvable. Other properties which hold for nilmanifolds, but not necessarily for solvmanifolds, are: 
1. If $M=G / \Delta$ is a nilmanifold, $G$ and $\Delta$ may be chosen so that $\Delta$ is discrete, hence $\pi=\Delta$.

2. Every homomorphism $\pi \rightarrow \pi$ extends to a homomorphism $G \rightarrow G$.

A connected, simply connected solvable Lie group for which exp : $L(G) \rightarrow$ $G$ is surjective is called an exponential (solvable) Lie group. The class of such groups will be denoted $\mathcal{E}$, and we will refer to solvmanifolds which can be represented as $G / \Delta, G \in \mathcal{E}$ as exponential solvmanifolds. Solvmanifolds for which $\pi$ can be embedded as a uniform subgroup of a connected simply connected solvable Lie group (i.e. for which $\pi=\Delta$ ) have been referred to in a variety of names by different authors. We will employ the language of [4] and refer to them as special solvmanifolds. Both classes of solvmanifolds can be characterized algebraically, in terms of the fundamental group $\pi$. The characterization of special solvmanifolds (which can be found in [1]) is involved, and not directly relevant to our work, so we will omit it. The description of exponential solvmanifolds, however, will be of some interest in our work. To give this description, we introduce a construction that will be of central importance in the development of Nielsen theory for solvmanifolds: the Mostow fibration.

Suppose $\pi$ is a finitely generated torsion-free $S$-group and $\Gamma \triangleleft \pi$ any nilpotent normal subgroup. Let $\Lambda=\pi / \Gamma$. The factorization $\Gamma \rightarrow \pi \rightarrow \Lambda$ can be realized as the fundamental group sequence of a fibration $N \rightarrow S \rightarrow$ $T$, with $N$ a manifold and $T$ a torus. We refer to such a fibration as a Mostow fibration. For us, there is one such Mostow fibration that is of particular importance. Let $\rho: \pi \rightarrow \pi^{*}$ be the abelianization of $\pi$. Decompose the abelianization $\pi^{*}$ as $F \oplus T$, with $F$ free abelian and $T$ finite, and let $\Gamma=\rho^{-1}(T)$. Since $\pi$ is a strongly torsion-free $S$-group, $\Gamma$ is nilpotent, and $\Lambda_{0}=\pi / \Gamma$ is clearly torsion-free abelian. Since $[\pi, \pi]$ is fully invariant, as is $T$ in the product $F \oplus T$, we see that $\Gamma$ is a fully invariant subgroup of $\pi$. Thus we have the following

Proposition 2.2 [14]. Let $S$ be a compact solvmanifold with $\pi=\pi_{1}(S)$. Suppose that $\Gamma$ is the (unique) nilpotent subgroup of $\pi$ such that $[\pi, \pi]$ is a subgroup of finite index in $\Gamma$ and $\Lambda_{0}=\pi / \Gamma$ is torsion free. Then there is a Mostow fibration $N \rightarrow S \rightarrow T_{0}$ in which $N$ is a nilmanifold with $\pi_{1}(N) \cong \Gamma$ and $T_{0}$ is a torus with $\pi_{1}\left(T_{0}\right)=\Lambda_{0}$. Furthermore, in every homotopy class of self maps on $S$ there is a fiber preserving map of this Mostow fibration.

This fibration is usually referred to as the minimal Mostow fibration, but as this is the only Mostow fibration we will consider in this work, we will simply refer to it as the Mostow fibration of $S$. The action of $\Lambda_{0}=\pi_{1}\left(T_{0}\right)$ on the fiber $N$ will be of particular importance to us. If $\lambda \in \Lambda_{0}$, then the 
lifting function can be chosen so that the fiber translation map $\tau_{\lambda}$ is given by a conjugation $c_{g}$ for some $g \in \pi$ that projects to $\lambda$. This does not provide a well-defined action of $\Lambda_{0}$ on $\Gamma$, but it does produce well-defined actions of $\Lambda_{0}$ on the $\Lambda_{i}, i \geq 1$. That is, conjugation by any element of $\pi$ preserves the $\Gamma_{i}$, and so produces an automorphism on $\Lambda_{i}$. But conjugation by elements of $\Gamma$ is trivial, so there is a well-defined action of $\Lambda_{0}$ on $\Lambda_{i}$. This action can be viewed as a homomorphism $A_{i}: \Lambda_{0} \rightarrow \operatorname{Aut}\left(\Lambda_{i}\right)$. We will refer to the collection $\left\{A_{i}\right\}_{i=1}^{n}$ as the linearization of the action of $\Lambda_{0}$ on $N$. The factor group $\Lambda_{0}$ of the Mostow fibration, together with the subquotients $\Lambda_{1}, \ldots, \Lambda_{k}$ of $\Gamma$ and the actions $\left\{A_{i}\right\}_{i=1}^{n}$ of $\Lambda_{0}$ on $\Lambda_{i}$ together constitute the linearization of $S,\left\{\Lambda_{i}, A_{i}\right\}$.

Exponential solvmanifolds can be easily characterized in terms of this linearization (cf. [2]). Suppose $S$ is a solvmanifold with linearization $\left\{\Lambda_{i}, A_{i}\right\}$. Then $S$ is an exponential solvmanifold if and only if no $A_{i}(\lambda)$ has an eigenvalue other than 1 on the unit circle. We will refer to a solvmanifold as an $\mathcal{N} R$-solvmanifold (for "no roots") if no $A(\lambda)$ has a root of unity other than 1 as an eigenvalue. Clearly, all exponential solvmanifolds are $\mathcal{N} R$ solvmanifolds.

However, not all $\mathcal{N} R$-solvmanifolds are exponential solvmanifolds. For example, consider the semi-direct product $\pi=\mathbb{Z}^{8} \rtimes \mathbb{Z}$, with $\mathbb{Z}$ acting on $\mathbb{Z}^{8}$ by

$$
A=\left[\begin{array}{cccccccc}
0 & 1 & 0 & 0 & 0 & 0 & 0 & 0 \\
0 & 0 & 1 & 0 & 0 & 0 & 0 & 0 \\
0 & 0 & 0 & 1 & 0 & 0 & 0 & 0 \\
0 & 0 & 0 & 0 & 1 & 0 & 0 & 0 \\
0 & 0 & 0 & 0 & 0 & 1 & 0 & 0 \\
0 & 0 & 0 & 0 & 0 & 0 & 1 & 0 \\
0 & 0 & 0 & 0 & 0 & 0 & 0 & 1 \\
-1 & 0 & 12 & 0 & -6 & 0 & 12 & 0
\end{array}\right] .
$$

The eigenvalues of $A$ are $\pm \alpha, \pm \frac{1}{\alpha}, \pm \beta, \pm \frac{1}{\beta}$, where $\alpha=\sqrt{2-\sqrt{2}}+i \sqrt{\sqrt{2}-1}$ and $\beta=\sqrt{2+\sqrt{2}}+\sqrt{\sqrt{2}+1}$. None of these are roots of unity, but $|\alpha|=1$. The solvmanifold with fundamental group $\pi$ is then an example of an $\mathcal{N} R$ solvmanifold which is not an exponential solvmanifold.

Just as a solvmanifold $S$ has a linearization, so too does a self-map on $S$. If $f: S \rightarrow S$ is a self-map, then we may assume (up to homotopy) that $f$ is a fiber-preserving map of the Mostow fibration, covering a homomorphism $\bar{f}_{0}: T_{0} \rightarrow T_{0} . \bar{f}_{0}$ has a corresponding homomorphism $F_{0}: \Lambda_{0} \rightarrow \Lambda_{0}$. Since $0 \in T_{0}$ is fixed by $\bar{f}_{0}$, the fiber $N_{0}$ over 0 is invariant under $f$. As a self-map of a nilmanifold, $f \mid N_{0}$ then has a linearization $\left\{\bar{F}_{i}\right\}_{i=1}^{k}$. If $\lambda \in \Lambda_{0}$, then $\left(f \mid N_{0}\right) \circ \tau_{\lambda} \simeq \tau_{F_{0} \lambda} \circ\left(f \mid N_{0}\right)$. This implies that $F_{i} \circ A_{i}(\lambda)=A_{i}\left(F_{0} \lambda\right) \circ F_{i}$ for 
all $i=1, \ldots, k$. The linearization of self-map on $S$, then, is a collection of homomorphisms $\left\{F_{i}: \Lambda_{i} \rightarrow \Lambda_{i}\right\}_{i=0}^{k}$ such that $F_{i} \circ A_{i}(\lambda)=A_{i}\left(F_{0} \lambda\right) \circ F_{i}$ for all $i=1, \ldots, k$.

We can make these linearizations even more concrete. Let $n_{i}=\operatorname{dim}\left(T_{i}\right)$. Then $\Lambda_{i} \cong \mathbb{Z}^{n_{i}}$; the action of $\Lambda_{0}$ on $\Lambda_{i}$ is given by a homomorphism $A_{i}$ : $\mathbb{Z}^{n_{0}} \rightarrow S L_{n_{i}}(\mathbb{Z})$; and the linearization of $F: S \rightarrow S$ is given by a collection of maps $F_{i} \in g l_{n_{i}}(\mathbb{Z})$ such that $F_{i} A_{i}(v)=A_{i}\left(F_{0} v\right) F_{i}$. We will see in the next section that these linearizations of solvmanifolds and self-maps on solvmanifolds carry all of the information needed for Nielsen theory.

\section{Nielsen and Lefschetz numbers.}

Having established the necessary algebraic-topological properties of the spaces involved, we now turn to a brief exposition of Nielsen theory, and its application to nilmanifolds and solvmanifolds.

Suppose that $f: M \rightarrow M$ is a map on a compact manifold. Then the Nielsen number $N(f)$ is defined in two steps as follows. (For more details see [11].) First, the set of fixed points of $f, \operatorname{Fix}(f)$, is partitioned into Nielsen classes by the relation that $x \sim_{f} y$ if there is a path $c$ from $x$ to $y$ with $c \simeq f c$. Now each Nielsen fixed point class $\mathbf{F} \subseteq \operatorname{Fix}(f)$ is both compact and open in $\operatorname{Fix}(f)$ and thus has an integer valued index, index $\operatorname{x}_{M}(f, \mathbf{F})$, defined. The Nielsen number $N(f)$ is then defined as the number of essential Nielsen classes, i.e. those with nonzero index. $N(f)$ is a homotopy invariant as well as a lower bound for $|\operatorname{Fix}(g)|$ for any $g \sim f$. For all compact manifolds save surfaces with negative Euler characteristic, any inessential class can be homotoped away and any essential class reduces to a singleton. Since all infrasolvmanifolds have $\chi(M)=0, N(f)=\min \{|\operatorname{Fix}(g)|: g \simeq f\}$ for all selfmaps on infrasolvmanifolds.

The Lefschetz number, in contrast, can be defined as the sum of the indices of the Nielsen fixed point classes. The natural route to establishing the equality $N(f)=|L(f)|$ is to show that every Nielsen class has the same index, and that that index is either $-1,0$ or +1 . This is precisely what is done in [7] for torus maps. Let $T=\mathbb{R}^{n} / \mathbb{Z}^{n}$ be the torus of dimension $n$. Given $f: T \rightarrow T$, the asphericity of $T$ makes it possible to assume, up to homotopy, that $f$ is covered by a linear map $F: \mathbb{R}^{n} \rightarrow \mathbb{R}^{n}$ whose matrix with respect to the usual basis has integer entries. Then every Nielsen fixed point class has the same index, which is either $-1,0$, or +1 , and $L(f)=\operatorname{det}(F-I)$. Thus for torus maps, $N(f)=|L(f)|=|\operatorname{det}(F-I)|$. Note that $N(f)=0$ if and only if 1 is an eigenvalue of $F$. In this case, an arbitrarily small perturbation of $f$ will be fixed point free. If 1 is not an eigenvalue, then every point of Fix $(f)$ will be an isolated fixed point and a Nielsen fixed point class. All of 
these will have a fixed point index of \pm 1 .

For self-maps of nilmanifolds, the orientable fibrations

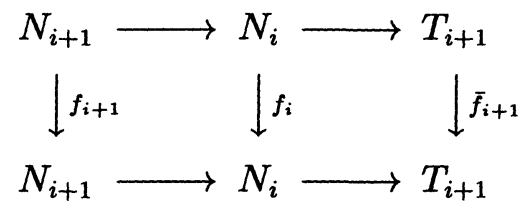

carry Nielsen and Lefschetz number product theorems, i.e.

$$
\begin{gathered}
\left|L\left(f_{i}\right)\right|=\left|L\left(f_{i}\right)\right|\left|L\left(\bar{f}_{i+1}\right)\right| \\
N\left(f_{i}\right)=N\left(f_{i}\right) N\left(\bar{f}_{i+1}\right) .
\end{gathered}
$$

From these, it follows that

$$
N(f)=\prod_{i=1}^{k} N\left(\bar{f}_{i}\right)=\prod_{i=1}^{k}\left|\operatorname{det}\left(F_{i}-I\right)\right|=\prod_{i=1}^{k}\left|L\left(\bar{f}_{i}\right)\right|=|L(f)| .
$$

For self maps on solvmanifolds, the Mostow fibration is nonorientable, so the product formulas do not immediately obtain. Instead, a more general pattern is observed. As noted above, we have for any homotopy class of maps on a solvmanifold $S$ a commutative diagram

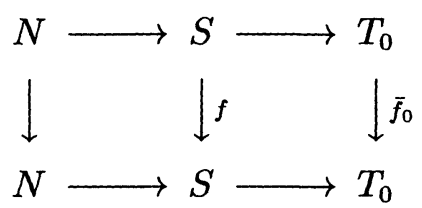

with $\bar{f}_{0}$ a torus endomorphism. If $N\left(\bar{f}_{0}\right)=0$, then $\bar{f}_{0}$ is homotopic to a fixed point free map. This homotopy lifts to a homotopy on $S$ which perturbs $f$ to a fixed point free map. Then it is trivial that $N(f)=|L(f)|=0$. We will therefore assume henceforth that $N\left(\bar{f}_{0}\right) \neq 0$. In this case, each point in $\operatorname{Fix}\left(\bar{f}_{0}\right)$ forms a distinct Nielsen fixed point class (hence $\operatorname{Fix}\left(\bar{f}_{0}\right)$ is a finite subset of $T_{0}$ ), and every Nielsen class of $f$ lies in a distinct fiber.

For $b \in \operatorname{Fix}\left(\bar{f}_{0}\right)$ let $N_{b}$ denote the fiber over $b$ and $f_{b}=f \mid N_{b}: N_{b} \rightarrow N_{b}$. To avoid notational confusion, denote the map on the fiber over $0 \in T_{0}$ by $f_{e}$. The result of the analysis in [14] is that

$$
\begin{aligned}
N(f) & =\sum_{b \in \operatorname{Fix}\left(\bar{f}_{0}\right)} N\left(f_{b}\right)=\sum_{b \in \operatorname{Fix}\left(\bar{f}_{0}\right)}\left|L\left(f_{b}\right)\right| \\
|L(f)| & =\left|\sum_{b \in \operatorname{Fix}\left(\bar{f}_{0}\right)} L\left(f_{b}\right)\right| .
\end{aligned}
$$


Thus we will have the equality $N(f)=|L(f)|$ if and only if every $L\left(f_{b}\right)$ has the same sign. Since the Mostow fibration is nonorientable, the fiber maps $f_{b}$ need not have the same homotopy type. The next step is therefore to determine how these maps are related to one another.

Suppose that $c$ is a path from 0 to a point $b \in \operatorname{Fix}\left(\bar{f}_{0}\right)$. Then in terms of fiber translation maps, $f_{b} \circ \tau_{c} \simeq \tau_{\bar{f}_{0} c} \circ f_{e}$, or $f_{b} \simeq \tau(c) \tau\left(c^{-1} * \bar{f}_{0} c\right) f_{e} \tau\left(c^{-1}\right)$. Thus if $\left\{F_{i}\right\}$ is the linearization of $f_{e}$, then the linearization of $f_{b}$ is (up to conjugation), $\left\{A_{i}\left(\omega_{b}\right) F_{i}\right\}$, where $\omega_{b}=c^{-1} * \bar{f}_{0} c \in \pi_{1}\left(T_{0}, 0\right)=\Lambda_{0}$. Note that the homotopy type of $f_{b}$ will clearly be different than that of $f_{e}$ when $\left\{A_{i}\left(\omega_{b}\right) F_{i}\right\}$ is different than $\left\{F_{i}\right\}$. The nonorientability of our fibration makes the action of the base on the fiber nontrivial so we could expect that this is often the case. We can rewrite the Nielsen and Lefschetz formulas as

$$
\begin{aligned}
N(f) & =\sum_{b \in \operatorname{Fix}\left(\bar{f}_{0}\right)} \prod_{i=1}^{k}\left|\operatorname{det}\left(A_{i}\left(\omega_{b}\right) F_{i}-I\right)\right| \\
|L(f)| & =\left|\sum_{b \in \operatorname{Fix}\left(\bar{f}_{0}\right)} \prod_{i=1}^{k} \operatorname{det}\left(A_{i}\left(\omega_{b}\right) F_{i}-I\right)\right| .
\end{aligned}
$$

Clearly, $N(f)=|L(f)|$ whenever the determinants $\operatorname{det}\left(A_{i}(\omega) F_{i}-I\right)$ are independent of $\omega \in \Lambda_{0}$ for all $i$. In fact, when this happens, we get, despite the previously mentioned variance in homotopy, the even stronger result of uniformity of $L\left(f_{b}\right)$.

Theorem 3.1. Suppose that $f: S \rightarrow S$ is a self map of an $\mathcal{N} R$-solvmanifold $S$, with linearization $\left\{F_{i}\right\}_{i=1}^{k}$. Then the product formula

$$
N(f)=\prod_{i=1}^{k} N\left(\bar{f}_{i}\right)=\prod_{i=1}^{k}\left|\operatorname{det}\left(F_{i}-I\right)\right|=\prod_{i=1}^{k}\left|L\left(\bar{f}_{i}\right)\right|=|L(f)|
$$

holds.

Thus the proof of this theorem has been reduced to a problem in linear algebra: given $F_{0} \in g l_{n_{0}}(\mathbb{Z})$ which does not have 1 as an eigenvalue, $F_{i} \in$ $g l_{n_{i}}(\mathbb{Z})$ and $A_{i}: \mathbb{Z}_{0}^{n} \rightarrow S L_{n_{i}}(\mathbb{Z})$ such that $F_{i} A_{i}(v)=A_{i}\left(F_{0} v\right) F_{i}$ and no $A_{i}(v)$ has a root of unity other than 1 as an eigenvalue, show that $\operatorname{det}\left(A_{i}(v) F_{i}-I\right)$ is independent of $v$ (i.e. that $\operatorname{det}\left(A_{i}(v) F_{i}-I\right)=\operatorname{det}\left(F_{i}-I\right)$ for all $v$ ). This matrix analysis is undertaken in the next section.

\section{Matrix Analysis.}

In this section, we will consider the following setting: $X \in g l_{n}(\mathbb{Z}), A: \mathbb{Z}^{m} \rightarrow$ $S L_{n}(\mathbb{Z})$, and $\Phi \in g l_{m}(\mathbb{Z})$ such that $X A(v)=A(\Phi v) X$ for all $v \in \mathbb{Z}^{m}$. We 
will refer to this assumption by saying that $X \Phi$-commutes with $A(v)$. The goal of this section is to prove the following

Theorem 4.1. If no $A(v)$ has a root of unity other than 1 as an eigenvalue and $\Phi$ does not have 1 as an eigenvalue, then $\operatorname{det}(A(v) X-I)$ is independent of $v$; that is, $\operatorname{det}(A(v)-I)=\operatorname{det}(X-I)$ for all $v \in \mathbb{Z}^{n}$.

First, consider the map $A: \mathbb{Z}^{m} \rightarrow S L_{n}(\mathbb{Z})$. We can view this as choosing a collection of commuting $n \times n$ integer matrices $A_{1}, \ldots, A_{m}$ with integer inverses. Then if $v \in \mathbb{Z}^{m}$ is given as $v=\left(v_{1}, \ldots, v_{m}\right)$, we can write $A(v)$ as $A_{1}^{v_{1}} \ldots A_{m}^{v_{m}}$. If $\lambda_{i}$ is an eigenvalue of $A_{i}$, let $E_{i}\left(\lambda_{i}\right)$ be the corresponding generalized eigenspace, and let $E\left(\lambda_{1}, \ldots, \lambda_{m}\right)=E_{1}\left(\lambda_{1}\right) \cap \cdots \cap E_{m}\left(\lambda_{m}\right)$. Since the matrices $A_{1}, \ldots, A_{m}$ commute, $\mathbb{R}^{n}$ has a direct-sum decomposition into the $E\left(\lambda_{1}, \ldots, \lambda_{m}\right)$, as $\lambda_{1}, \ldots, \lambda_{m}$ range over the spectra of $A_{1}, \ldots, A_{m}$ respectively. Since every $A_{i}$ leaves all $E\left(\lambda_{1}, \ldots, \lambda_{m}\right)$ invariant, every $A(v)$ likewise leaves all of them invariant, and has the single eigenvalue $\lambda_{1}^{v_{1}} \cdots \lambda_{m}^{v_{m}}$ on $E\left(\lambda_{1}, \ldots, \lambda_{m}\right)$.

This gives in a natural way the semisimple-unipotent decompositions of the matrices $A(v)$. Let $T(v)$ be the transformation such that $T(v)=$ $\lambda_{1}^{v_{1}} \cdots \lambda_{m}^{v_{m}} I$ on $E\left(\lambda_{1}, \ldots, \lambda_{m}\right)$ and let $U(v)=A(v) T(v)^{-1}$. Then all transformations in the collection $\{T(v), U(v)\}$ commute with one another; all the $T(v)$ 's are semisimple and all of the $U(v)$ 's are unipotent. Of course, this is the unique decomposition with these properties.

Now, let us consider the transformation $X$ and the relation $X A(v)=$ $A(\Phi v) X$.

Lemma 4.2. If $X A=B X$, then $X$ maps $\operatorname{ker}(A-\lambda I)^{k}$ into $\operatorname{ker}(B-\lambda I)^{k}$, for every $\lambda \in \mathbb{C}$ and $k \geq 1$.

Lemma 4.3. If $X A(v)=A(\Phi v) X$ and $A(v)=T(v) U(v)$ is the semisimpleunipotent decomposition of $A(v)$, then $X T(v)=T(\Phi v) X$ and $X U(v)=$ $U(\Phi v) X$ for all $v$.

Proof. Since $X$ maps the $\lambda$ generalized eigenspace of $A(v)$ to the $\lambda$ generalized eigenspace of $A(\Phi v), X T(v)=\lambda X=T(\Phi v) X$ on the $\lambda$ generalized eigenspace of $A(v)$. Thus $X T(v)=T(\Phi v) X$. Since $U(v)=A(v) T(v)^{-1}$ and both $A(v)$ and $T(v) \Phi$-commute with $X$, it follows immediately that $U(v)$ also $\Phi$-commutes with $X$.

We will be interested in the quantity $\operatorname{det}(A(v) X-I)$, and in particular, we will be interested in discovering when this quantity is independent of $v$. We begin with a few obvious assertions. 
Lemma 4.4. If $\left\{e_{j}\right\}$ is a basis for $\mathbb{Z}^{m}$ and $\operatorname{det}\left(A\left(e_{j}\right) X-I\right)=\operatorname{det}(X-I)$ for all $j$, then $\operatorname{det}(A(v) X-I)=\operatorname{det}(X-I)$ for all $v \in \mathbb{Z}^{m}$.

Lemma 4.5. The quantity $\operatorname{det}(A(v)-I)$ is constant on orbits of $\Phi$. That $i s, \operatorname{det}\left(A\left(\Phi^{k} v\right) X-I\right)=\operatorname{det}(A(v) X-I)$ for all $k$.

This allows us (among other things) to assume without loss of generality that $\Phi$ does not have 0 as an eigenvalue. Let $V_{0}$ be the generalized 0 eigenspace of $\Phi$ and let $V_{1}=\operatorname{Im}\left(\Phi^{m}\right)$. Then $\mathbb{R}^{m}=V_{0} \oplus V_{1}$, and $\mathbb{Z}^{m}=\left(V_{0} \cap\right.$ $\left.\mathbb{Z}^{m}\right) \bigoplus\left(V_{1} \cap \mathbb{Z}^{m}\right)$. Clearly, for every $v \in \mathbb{Z}^{m}, \Phi^{m}(v) \in V_{1}$, and $\operatorname{det}(X A(v)$ $I)=\operatorname{det}\left(X A\left(\Phi^{m}(v)\right)-I\right)$. Moreover, $\Phi$ leaves $V_{1}$ invariant, and does not have 0 as an eigenvalue on $V_{1}$. So if we restrict $\Phi$ to $V_{1}$ and show that $\operatorname{det}(X A(v)-I)$ is independent of $v$ on $V_{1}$, then it will be independent of $v$ on $\mathbb{R}^{m}$. We will assume for the rest of this section that 0 is not an eigenvalue of $\Phi$.

We will prove theorem 4.1 by first reducing to the separate cases where the action matrices $A(v)$ are either all unipotent or all semisimple.

Lemma 4.6. If $\operatorname{det}(T(v) X-I)$ and $\operatorname{det}(U(v) X-I)$ are independent of $v$ for all $X$ which $\Phi$-commute with $T(v)$ and $U(v)$, then $\operatorname{det}(A(v) X-I)$ is independent of $v$ for all $X$ which $\Phi$-commute with $A(v)$.

Proof. Since $T(v)$ commutes with $U(v)$ and $X \Phi$-commutes with $U(v)$, their product $T(v) X \Phi$-commutes with $U(v)$. Thus

$$
\operatorname{det}(A(v) X-I)=\operatorname{det}(T(v) U(v) X-I)=\operatorname{det}(T(v) X-I)=\operatorname{det}(X-I) .
$$

The unipotent case is considerably easier, and we will dispose of it first.

Lemma 4.7. If $\Phi$ does not have 0 or 1 as an eigenvalue, $U: \mathbb{Z}^{m} \rightarrow S L_{n}(\mathbb{Z})$ is a homomorphism whose image consists of unipotent matrices $U(v)$, and $X$ is a matrix which $\Phi$-commutes with $U(v)$, then $\operatorname{det}(U(v) X-I)$ is independent of $v$.

Proof. Proceed inductively on $n$. For $n=1$, each $U(v)=i d$ and the statement is trivial.

For the general case, let

$$
K=\bigcap_{v \in \mathbb{Z}^{m}} \operatorname{ker}(U(v)-I)
$$

and let

$$
K_{\Phi}=\bigcap_{v \in \mathbb{Z}^{m}} \operatorname{ker}(U(\Phi v)-I)
$$


Note that, since the matrices commute, they preserve each others 1eigenspaces, so $K \neq 0$. Then $0 \neq K \subset K_{\Phi}$, and $X(K) \subset K_{\Phi}$. In fact, $K=K_{\Phi}$. That is, since $\Phi$ is injective, $\Phi\left(\mathbb{Z}^{m}\right)$ has finite index in $\mathbb{Z}^{m}$. In particular, if $v \in \mathbb{Z}^{m}$, there is some $p$ such that $p v \in \Phi\left(\mathbb{Z}^{m}\right)$. If $w \in K_{\Phi}$, then $w=U(p v) w=U(v)^{p} w$. But since $U(v)$ is unipotent, this implies that $U(v) w=w$. Thus $X$ and all $U(v)$ 's leave $K$ invariant.

Now let $L=\mathbb{R}^{n} / K$ and let $X_{1}: K \rightarrow K$ and $X_{2}: L \rightarrow L$ be the restriction and quotient of $X$ respectively. Similarly, let $U_{1}(v): K \rightarrow K$ and $U_{2}(v): L \rightarrow L$ be the factorization of $U(v)$. Then $\operatorname{det}(U(v) X-I)=$ $\operatorname{det}\left(U_{1}(v) X_{1}-I_{K}\right) \operatorname{det}\left(U_{2}(v) X_{2}-I_{L}\right)$. On $K$, all $U_{1}(v)=i d$, so it is trivial that $\operatorname{det}\left(U_{1}(v) X_{1}-I_{K}\right)$ is independent of $v$. By induction, $\operatorname{det}\left(U_{2}(v) X_{2}-I_{L}\right)$ is independent of $v$, so $\operatorname{det}(U(v) X-I)$ is as well.

Lemma 4.8. If $\Phi$ does not have 0 or 1 as an eigenvalue, $T: \mathbb{Z}^{m} \rightarrow S L_{n}(\mathbb{Z})$ is a homomorphism whose image consists of semisimple matrices $T(v)$ which have no roots of unity (other than 1 ) as eigenvalues, and $X$ is a matrix which $\Phi$-commutes with $T(v)$, then $\operatorname{det}(T(v) X-I)$ is independent of $v$.

To prove this lemma, and so complete the proof of theorem 4.1, we will need to make careful use of the relationship between the eigenvalues of the matrices $T(v)$ (which cannot be roots of unity other than 1) and the eigenvalues of $\Phi$ (which cannot be 0 or 1 ). The first step will be to reformulate the problem to fully display this relationship. To do so, choose a basis $\left\{e_{j}\right\}$ for $\mathbb{Z}^{m}$ and a basis of common eigenvectors $\left\{f_{j}\right\}$ of the operators $T(v)$ on $\mathbb{R}^{n}$. To avoid overburdening the notation, we adopt the following convention. Suppose $\sigma \in S_{n}$ has a cycle of length $s(1 \leq s \leq n)$. Choose an integer $h$ in that cycle and denote $\sigma^{i}(h)$ by $\sigma(i)$. Let $\lambda_{i}(v)$ be the eigenvalue of $T(v)$ on $f_{\sigma(i)}$, and let $\lambda_{i, j}=\lambda_{i}\left(e_{j}\right)$. Let $Y=\left[y_{i j}\right]$ be the matrix of $X-I$ in terms of the basis $\left\{f_{i}\right\}$. Then from the standard theory of determinants and the fact that every permutation factors into a product of cyclic ones, we have

Proposition 4.9. Every term in $\operatorname{det}(T(v) X-I)$ is a product of factors of the form

$$
\prod_{i=1}^{s} \lambda_{i}(v) y_{\sigma(i) \sigma(i+1)}
$$

where $\sigma$ is a cyclic permutation of order s. Such a factor is nonzero only if $\lambda_{i}(\Phi w)=\lambda_{i+1}(w)$ for all $i$ and all $w$.

Note: we will show the independence on $v$ by proving that $\prod_{i=1}^{s} \lambda_{i}(v)=1$. Except for the diagonal entries (i.e. when $s=1$ ) the matrix for $X-I$ is the same as the matrix for $X$. In this case we will thus see that $\lambda_{i}(v)=1$ as needed to give the above predicted form. 
Thus, for the remainder of this section, in order to make our computation more concrete we fix $s$ and define a specific basis $\Delta=\left\{e_{j}\right\}_{j=1}^{m}$ for $\mathbb{Z}$ as follows. The $e_{i}$ are to be chosen so that $\left\{e_{1}, \ldots, e_{l}\right\}(1 \leq l \leq m) \operatorname{span} K=\operatorname{ker}\left(\Phi^{s}-I\right)$ and $J=\operatorname{span}\left\{e_{l+1}, \ldots, e_{m}\right\}$ meets $K$ only trivially. This is possible since $\Phi^{s}-I$ has a representation as an integer matrix so that $K \cap \mathbb{Z}^{m}$ is uniform in $K$.

If $\Phi$ is expressed as $\left[\phi_{i j}\right]$ in the basis $\left\{e_{j}\right\}$, the condition for a factor to be nonzero is equivalent to

$$
\lambda_{i+1, j}=\lambda_{i}\left(\left(\phi_{1 j}, \ldots, \phi_{m j}\right)\right)=\prod_{k=1}^{m} \lambda_{i, k}^{\phi_{k j}} \quad \forall i, j .
$$

Write $\lambda_{i, j}=\exp \left(r_{i, j}+2 \pi i \theta_{i, j}\right)$, with $\theta_{i, j}$ defined $\bmod 1$. The condition then becomes

$$
\exp \left(r_{i+1, j}+2 \pi i \theta_{i+1, j}\right)=\exp \left(\sum_{k=1}^{m}\left(\phi_{k j} r_{i, k}+2 \pi i \phi_{k j} \theta_{i, k}\right)\right)
$$

or

$$
\begin{aligned}
r_{i+1, j} & =\sum_{k=1}^{m} \phi_{k j} r_{i, k} \\
\theta_{i+1, j} & =\sum_{k=1}^{m} \phi_{k j} \theta_{i, k} \bmod 1 .
\end{aligned}
$$

Now, let $R_{i}, \Theta_{i} \in \mathbb{R}^{m}$ be the vectors whose $j$ th components are $r_{i, j}$ and $\theta_{i, j}$ respectively. The equations then become

$$
\begin{gathered}
R_{i+1}=\Phi^{T} R_{i} \\
\Theta_{i+1}=\Phi^{T} \Theta_{i} \bmod \mathbb{Z}^{m} .
\end{gathered}
$$

Our goal is to prove that $\operatorname{det}\left(X T\left(e_{j}\right)-I\right)=\operatorname{det}(X-I)$ for all $j$. This can be done by showing that $\prod_{i=1}^{s} \lambda_{i, j}=1$ for every $j$ whenever (1) is satisfied. In our $(r, \theta)$ notation, $\prod_{i=1}^{s} \lambda_{i, j}=\exp \left(\sum_{i=1}^{s} r_{i, j}+2 \pi i \theta_{i, j}\right)$, so we must show $\sum_{i=1}^{s} r_{i, j}=0$ and $\sum_{i=1}^{s} \theta_{i, j} \in \mathbb{Z}$ for every $j$. That is, we must show $\sum_{i=1}^{s} R_{i}=0$ and $\sum_{i=1}^{s} \Theta_{i} \in \mathbb{Z}^{m}$. In other words, we have reduced the problem to proving the following in the basis $\Delta$.

Proposition 4.10. Suppose $\Phi \in G L_{m}(\mathbb{Z})$ does not have 1 as an eigenvalue, and suppose $R_{1}, \ldots, R_{s} \in \mathbb{R}^{m}$ and $\Theta_{1}, \ldots, \Theta_{s} \in \mathbb{R}^{m}$ (defined modulo $\mathbb{Z}^{m}$ ) are vectors such that

1. $R_{i+1}=\Phi R_{i}$ and $\Theta_{i+1}=\Phi \Theta_{i} \bmod \mathbb{Z}^{m}($ with $i$ counted $\bmod s)$. 
2. If $r_{i, j}=0$, then $\theta_{i, j}$ is either irrational or 0 .

Then $\sum_{i=1}^{s} R_{i}=0$ and $\sum_{i=1}^{s} \Theta_{i} \in \mathbb{Z}^{m}$.

Proof. The assumption that $R_{i+1}=\Phi R_{i}$ means that $\Phi^{s} R_{i}=R_{i}$ for every $i$. Similarly, the assumption that $\Theta_{i+1}=\Phi \Theta_{i} \bmod \mathbb{Z}^{m}$ means that $\Theta_{i}=$ $\Phi^{s} \Theta_{i} \bmod \mathbb{Z}^{m}$ for every $i$. That is, each $R_{i} \in \operatorname{ker}\left(\Phi^{s}-I\right)$ so $r_{i, j}=0$ for $j>l$. This implies that $\theta_{i, j}$ is either irrational or 0 for $j>l$. Let $\Phi^{\prime}$ be the submatrix of $\Phi^{s}-I$ which maps $J$ to $J$, and let $\Theta_{i}{ }^{\prime}$ be the $J$-component of $\Theta_{i}$. Then the $J$-component of $\left(\Phi^{s}-I\right) \Theta_{i}$ is given by $\Phi^{\prime} \Theta_{i}{ }^{\prime}$, so $\Phi^{\prime} \Theta_{i}{ }^{\prime} \in \mathbb{Z}^{m}$. Since $\Phi^{\prime}$ is an invertible integer matrix, this implies that all components of $\Theta_{i}{ }^{\prime}$ are rational, hence $\Theta_{i}{ }^{\prime}=0$.

That is, we may assume that all $R_{i}$ and $\Theta_{i}$ lie in $K$, or equivalently, we may assume that $\Phi^{s}=I$. In particular, we can now choose the representatives $\Theta_{2}, \ldots, \Theta_{s}$ so that $\Theta_{i+1}=\Phi \Theta_{i}$ (again, with $i$ counted $\bmod s$ ). Now, it is easily seen that $\Phi\left(\sum_{i=1}^{n} R_{i}\right)=\sum_{i=1}^{n} R_{i}$ and $\Phi\left(\sum_{i=1}^{s} \Theta_{i}\right)=\sum_{i=1}^{n} \Theta_{i}$. Since $\Phi$ does not have 1 as an eigenvalue, $\sum_{i=1}^{s} R_{i}=\sum_{i=1}^{s} \Theta_{i}=0$.

\section{Concluding remarks.}

The full strength of our result, theorem 3.1, is not just the equality $N(f)=$ $|L(f)|$ for self maps on $\mathcal{N} R$-solvmanifolds, but the product theorem $N(f)=$ $\Pi N\left(\bar{f}_{i}\right)=\prod\left|\operatorname{det}\left(F_{i}-I\right)\right|$. This product formula was not established by using any of the standard product formula theorems, such as those in [18], but by direct calculation. The important difference between our result and most product formula results is that we do not assume (and indeed, do not conclude) that the maps on different fibers are homotopic. That is, we are using the strong algebraic information available to us to conclude that $N\left(f_{b}\right)$ is constant, even when the homotopy type $\left[f_{b}\right]$ is not.

We have established that $N(f)=|L(f)|$ for all self maps on a large class of solvmanifolds. On the other hand, there are examples of solvmanifolds (e.g. the Klein bottle) for which it is not true that $N(f)=|L(f)|$ for all maps [5]. It is therefore reasonable to ask if we can classify which compact solvmanifolds (or, more generally, which compact aspherical manifolds) have $N(f)=|L(f)|$ for all $f: S \rightarrow S$. We expect that this classification (if ever found) will be stated solely in terms of the fundamental group of $S$. For example, it seems quite possible that a solvmanifold has $N(f)=|L(f)|$ for all $f: S \rightarrow S$ if and only if $S \in \mathcal{N} R$. To prove this, we would have to show that for every solvmanifold $S \notin \mathcal{N} R$, there exists an $f: S \rightarrow S$ with $N(f)>|L(f)|$. In considering the possibility of such a result, it is worth noting that we only used the linearization of $S \in \mathcal{N} R$ and $f: S \rightarrow S$ in proving theorem 1. A natural approach to the converse would be to show 
that, given a linearization $\left\{\Lambda_{i}, A_{i}\right\}$ where some $A_{i}(\lambda)$ has a root of unity as an eigenvalue, then there exists a collection $F_{i}$ such that $F_{i} A_{i}(\lambda)=A_{i}\left(F_{0} \lambda\right) F_{i}$ and $\operatorname{sgn}\left(A_{i}(\lambda) F_{i}-I\right)=-\operatorname{sgn}\left(F_{i}-I\right)$ for an odd number of $i$.

The problem with such an approach is that such a collection of maps $\left\{F_{i}\right\}$ may not be the linearization of a topological map $f: S \rightarrow S$. That is, since the linearizations neglect some of the algebraic information of the fundamental group, it is not always possible to reconstruct the fundamental group $\pi$ from $\left\{\Lambda_{i}, A_{i}\right\}$, nor to construct a map $f: S \rightarrow S$ from $\left\{F_{i}\right\}$. Thus characterizing solvmanifolds which admit counterexamples (i.e. maps with $N(f)>|L(f)|)$ will probably require using more complete information on $\pi$ and $f_{\#}: \pi \rightarrow \pi$ than just the linearizations.

Of course, the more general problem is that of computing $N(f)$ (and hence $M F(f))$. The formula $N(f)=|L(f)|=\prod_{i=0}^{k}\left|\operatorname{det}\left(F_{i}-I\right)\right|$ for maps on $\mathcal{N} R$-solvmanifolds not only allows us to compute $N(f)$ for such maps; it also leads the way to computing $N(f)$ for all maps on infrasolvmanifolds. An infrasolvmanifold $M$ can be defined as a manifold which admits a solvmanifold as a finite regular cover. In fact, all such manifolds admit a finite regular cover by an $\mathcal{N} R$-solvmanifold [2]. The techniques of [16] then show that $N(f)$ for $f: M \rightarrow M$ can be computed from the Nielsen coincidence numbers $N(\tilde{f}, p)$, where $S_{1}, S_{2}$ are $\mathcal{N} R$-solvmanifolds which are finite regular covers of $M, p: S_{1} \rightarrow S_{2}$ is a covering projection, and $\tilde{f}: S_{1} \rightarrow S_{2}$ ranges over all lifts of $f: M \rightarrow M$. Thus Nielsen numbers for self maps on infrasolvmanifolds will be computable in terms of Lefschetz (coincidence) numbers provided we can establish the equality $N(\tilde{f}, p)=|L(\tilde{f}, p)|$.

This is a special case of the more general problem of computing Nielsen coincidence numbers $N(f, g)$ for pairs of maps $f, g: S_{1} \rightarrow S_{2}$. This problem has been considered in $[\mathbf{6}, \mathbf{1 0}, \mathbf{1 5}]$. The most general result is that of [15], that $N(f, g) \geq|L(f, g)|$ when $S_{1}, S_{2}$ are compact orientable manifolds of the same dimension and $S_{2}$ is a solvmanifold, and that $N(f, g)=|L(f, g)|$ when we further assume that $S_{2}$ is a nilmanifold. In light of theorem 1, it seems reasonable to conjecture that $N(f, g)=|L(f, g)|$ when $S_{2}$ is an $\mathcal{N} R$ solvmanifold. We will consider this conjecture, and in particular the special case $\tilde{f}, p: S_{1} \rightarrow S_{2}$ needed to compute Nielsen numbers on infrasolvmanifolds, in a future work.

\section{References}

[1] L. Auslander, Discrete uniform subgroups of solvable Lie groups, Trans. Amer. Math. Soc., 99 (1961), 398-402.

[2] - An exposition of the structure of the solvmanifolds. I: Algebraic theory, Bull. Amer. Math. Soc., 79 (1973), 227-261. 
[3] L. Auslander, L. Green and F. Hahn, Flows on homogeneous spaces, Annals of Mathematics Studies, 53, Princeton University Press (1963).

[4] L. Auslander and F.E.A. Johnson, On a conjecture of C. T. C. Wall, J. London Math. Soc.(2), 14 (1976), 331-332.

[5] D.V. Anosov, The Nielsen number of maps of nil-manifolds, Russian Math. Surveys, 40 (1985), 149-150.

[6] R. Brooks and P. Wong, On changing fixed points and coincidences to roots, Proc. Amer. Math. Soc., 115 (1992), 527-534.

[7] R. Brooks, R. Brown, J. Pak and D. Taylor, Nielsen numbers of maps of tori, Proc. Amer. Math. Soc., 52 (1975), 398-400.

[8] E. Fadell and S. Husseini, On a theorem of Anosov on Nielsen numbers for nilvmanifolds, Nonlinear Functional Analysis and its Applications (Maratea, 1985), 47-53, NATO Adv. Sci. Inst. Ser. C: Math. Phys. Sci., 173, Reidel, Dordrecht-Boston, Mass. 1986.

[9] F.T. Farrell, L.E. Jones, Classical Aspherical Manifolds, CBMS Lecture Notes \# 75, Amer. Math. Soc., Providence, R.I. 1990.

[10] J. Jezierski, The Nielsen number product formula for coincidences, Fund. Math., 134 (1989), 183-212.

[11] B. Jiang, Lectures on Nielsen fixed point theory, Contemporary Mathematics, vol.14, Amer. Math. Soc., Providence, RI 1983.

[12] E. Keppelman, Periodic points on nilmanifolds and solvmanifolds, preprint.

[13] A. Mal'cev, On a class of homogeneous spaces, Amer. Math. Soc. Transl. (2), 39 (1951), 276-307.

[14] C. McCord, Nielsen numbers and Lefschetz numbers on solvmanifolds, Pacific J. Math., 147 (1991), 153-164.

[15] _ Lefschetz and Nielsen coincidence numbers on nilmanifolds and solvmanifolds, Topology Appl., 43 (1992), 249-261.

[16] , Estimating Nielsen numbers on infrasolvmanifolds, Pac. J. Math., 154 (1992), 345-368.

[17] G.D. Mostow, Factor spaces for solvable groups, Ann. of Math., 60 (1954), 1-27.

[18] C.-Y. You, Fixed point classes of a fiber map, Pac. J. Math., 100 (1982), 217-241.

Received August 21, 1992 and revised January 20, 1993.

Texas A \& M UNIVERsity

College Station, TX 77843

AND

UNIVERSITY OF CincinNati

CincinNati, OH 45221 - 0025

E-MAIL ADDRESS: CHRIS.MCCORD@UC.EDU 



\title{
PACIFIC JOURNAL OF MATHEMATICS
}

Founded by E. F. Beckenbach (1906-1982) and F. Wolf (1904-1989)

\section{EDITORS}

Sun-Yung Alice Chang (Managing Editor)

University of California

Los Angeles, CA 90095-1555

pacific@math.ucla.edu

F. Michael Christ

University of California

Los Angeles, CA 90095-1555

christ@math.ucla.edu

Thomas Enright

University of California

San Diego, La Jolla, CA 92093

tenright@ucsd.edu

Nicholas Ercolani

University of Arizona

Tucson, AZ 85721

ercolani@math.arizona.edu
Robert Finn

Stanford University

Stanford, CA 94305

finn@gauss.stanford.edu

Vaughan F. R. Jones

University of California

Berkeley, CA 94720

vfr@math.berkeley.edu

Steven Kerckhoff

Stanford University

Stanford, CA 94305

spk@gauss.stanford.edu
Martin Scharlemann

University of California

Santa Barbara, CA 93106

mgscharl@math.ucsb.edu

Gang Tian

Courant Institute

New York University

New York, NY 10012-1100

tiang@taotao.cims.nyu.edu

V. S. Varadarajan

University of California

Los Angeles, CA 90095-1555

vsv@math.ucla.edu

\section{SUPPORTING INSTITUTIONS}

\section{CALIFORNIA INSTITUTE OF TECHNOLOGY \\ NEW MEXICO STATE UNIVERSITY \\ OREGON STATE UNIVERSITY \\ STANFORD UNIVERSITY \\ UNIVERSITY OF ARIZONA \\ UNIVERSITY OF BRITISH COLUMBIA \\ UNIVERSITY OF CALIFORNIA \\ UNIVERSITY OF HAWAII}

\author{
UNIVERSITY OF MONTANA \\ UNIVERSITY OF NEVADA, RENO \\ UNIVERSITY OF OREGON \\ UNIVERSITY OF SOUTHERN CALIFORNIA \\ UNIVERSITY OF UTAH \\ UNIVERSITY OF WASHINGTON \\ WASHINGTON STATE UNIVERSITY
}

The supporting Institutions listed above contribute to the cost of publication of this Journal, but they are not owners or publishers and have no responsibility for its contents or policies.

Manuscripts must be prepared in accordance with the instructions provided on the inside back cover.

The Pacific Journal of Mathematics (ISSN 0030-8730) is published monthly except for July and August. Regular subscription rate: $\$ 215.00$ a year (10 issues). Special rate: $\$ 108.00$ a year to individual members of supporting institutions.

Subscriptions, orders for back issues published within the last three years, and changes of subscribers address should be sent to Pacific Journal of Mathematics, P.O. Box 4163, Berkeley, CA 94704-0163, U.S.A. Prior back issues are obtainable from Kraus Periodicals Co., Route 100, Millwood, NY 10546.

The Pacific Journal of Mathematics at the University of California, c/o Department of Mathematics, 981 Evans Hall, Berkeley, CA 94720 (ISSN 0030-8730) is published monthly except for July and August. Second-class postage paid at Berkeley, CA 94704, and additional mailing offices. POSTMASTER: send address changes to Pacific Journal of Mathematics, P.O. Box 6143, Berkeley, CA 94704-0163.

\author{
PUBLISHED BY PACIFIC JOURNAL OF MATHEMATICS at University of California, \\ Berkeley, CA 94720, A NON-PROFIT CORPORATION \\ This publication was typeset using AMS-LATEX, \\ the American Mathematical Society's TEX macro system. \\ Copyright (C) 1995 by Pacific Journal of Mathematics
}




\section{PACIFIC JOURNAL OF MATHEMATICS}

Volume $170 \quad$ No. $1 \quad$ September 1995

Generalized generalized spin models (four-weight spin models)

EIICHI BANNAI and ETSUKO BANNAI

Fine structure of the Mackey machine for actions of abelian groups with constant Mackey obstruction

SIEGFRIED ECHTERHOFF and JONATHAN ROSENBERG

The corestriction of valued division algebras over Henselian fields. I

YOON SUNG HWANG

The corestriction of valued division algebras over Henselian fields. II

YOON SUNG HWANG

The cohomology of expansive $\mathbb{Z}^{d}$-actions by automorphisms of compact, 105 abelian groups

ANATOLE KATOK and KLAUS SCHMIDT

The Anosov theorem for exponential solvmanifolds

EDWARD KEPPELMANN and CHRISTOPHER K. MCCORD

Projections of measures on nilpotent orbits and asymptotic multiplicities 161 of $K$-types in rings of regular functions. I

DONALD RAYMOND KING

On almost-everywhere convergence of inverse spherical transforms

Christopher Meaney and Elena Prestini

Characters of supercuspidal representations of $S L(n)$

Fiona AnNe Murnaghan

The cohomology of higher-dimensional shifts of finite type

KLAUS SCHMIDT

On Gorenstein surface singularities with fundamental genus $p_{f} \geqq 2$ which satisfy some minimality conditions

TADASHI TOMARU 